\title{
Resonant interactions in Bénard-Marangoni convection in cylindrical containers
}

\author{
B. Echebarría *, D. Krmpotić * and C. Pérez-García * \\ * Departamento de Física y Matemática Aplicada, Facultad de Ciencias, \\ Universidad de Navarra, 31080 Pamplona, Navarra, Spain. \\ * Departamento de Física, Universidad Nacional de La Plata, \\ La Plata, Argentina.
}

October 29, 2018

\begin{abstract}
Convection in a cylindrical container of small aspect ratio is studied. It is known that when, in addition to buoyancy forces, thermocapillarity effects are taken into account, resonant interactions of two modes may appear. In the case of 1:2 resonance amplitude equations are derived, showing the existence of a stable heteroclinic orbit and rotating waves, until now not observed experimentally.
\end{abstract}

Keywords: Convection, Hydrodynamic instability, bifurcations, nonlinearity.

P.A.C.S.: $44.25 .+\mathrm{f}, 47.20 . \mathrm{Dr}, 47.20 . \mathrm{Tg}$ 


\section{Introduction}

Since the works of Bénard [1] at the beginning of this century, thermal convection became one of the most studied pattern forming systems [2]. In Bénard-Marangoni (BM) experiments a fluid layer confined in a container open to the atmosphere is heated from below. When the difference of temperature between the top and the bottom surface (i.e. $\Delta T$ ) is bigger than a critical value convection appears. Pearson [3] showed that besides buoyancy forces, thermocapillarity effects provide another instability mechanism in this case.

For shallow layers buoyancy forces dominate, while for thick layers thermocapillarity is the main mechanism. Two different regimes appear depending on the liquid depth and the aspect ratio $a$, i.e., the ratio between the horizontal and the vertical dimensions of the vessel. For big aspect ratios $(a>20)$ regular patterns appear at the onset of convection (typically hexagons in BM convection). For small $a(a<10)$ the arising patterns are determined by the geometry of the vessel. In a theoretical work Rosenblat et al. studied the linear and weakly nonlinear problem with small aspect ratio for cylindrical [4] and square [5] containers. In the linear case buoyancy and thermocapillary forces were considered. But the extension to the nonlinear regime was only made for pure thermocapillary effects.

There are important experimental contributions in these two regimes. For big aspect ratio the most interesting are due to Koschmieder [6], Cerisier [7] and Schatz et al. [8]. For small aspect ratio Koschmieder and Prahl [9] performed experiments in square and cylindrical containers near convective threshold. Ondarcuhu et al. described a series of dynamical patterns that look very different in square [10] and in cylindrical containers [11].

We study BM convection in a cylindrical container of small aspect ratio in the usual case when both, buoyancy and thermocapillarity forces, act together. In order to study the convective regime time dependent amplitudes of the unstable modes are considered. Bifurcation theory shows [12 that in a system with $O(2)$ symmetry but with the reflection symmetry with respect to the middplane broken (like in BM convection or in Rayleigh-Bénard convection under non-Boussinesq conditions) not only the modulus, but also the relative phases of the amplitudes must be taken into account. Codimension two (CT) points, where two modes become simultaneously unstable are specially interesting because they can lead to a resonant interaction with a characteristic dynamical behaviour. A similar case has been studied in convection in spherical cells ( $\mathrm{SO}(3)$ symmetry) by Friedrich and Haken [13]. 
In Section 2 we present the basic equations and the boundary conditions governing the dynamics of the system. In Section 3 we perform a linear analysis and compare our results with the experimental ones. Section 4 is devoted to the weakly nonlinear regime. In particular, we derive the amplitude equations for a 1:2 resonance, which were also derived for a model equation by means of bifurcation theory [14] and studied in detail in ref. [15]. A physical interpretation of these results is made. Finally, we discuss the main conclusions in Section 5.

\section{Evolution equations and boundary conditions}

Consider a fluid layer of depth $d$ confined between a conducting plate heated from below and an upper surface open to the atmosphere, under a temperature difference $\Delta T$. We assume that the fluid is Newtonian and that Boussinesq approximation holds. (We will follow the standard notation for the parameters of the fluid).

Then, in nondimensional variables (obtained dividing space, time, velocity and temperature by $d, d^{2} / \kappa, \kappa / d$ and $\Delta T$ respectively) the equations governing the dynamics of the fluid are:

$$
\begin{aligned}
& \nabla \cdot \mathbf{v}=0 \\
& \operatorname{Pr}^{-1}\left[\frac{\partial \mathbf{v}}{\partial t}+(\mathbf{v} \cdot \nabla) \mathbf{v}\right]=-\nabla p+R T \mathbf{e}_{\mathbf{z}}+\nabla^{2} \mathbf{v} \\
& \frac{\partial T}{\partial t}+\mathbf{v} \cdot \nabla T=\nabla^{2} T
\end{aligned}
$$

where the Prandtl number $\operatorname{Pr}=\nu / \kappa$ gives an estimation of the relative importance of thermal and mechanical dissipative processes. In this work we take the limit $\operatorname{Pr} \rightarrow \infty$, equivalent to assume that velocity perturbations relax much faster than temperature perturbations. This limit allows to neglect both, the advective term and the temporal derivative of the velocity in the Navier-Stokes equation, so the velocity field follows adiabatically the variations of temperature. The Rayleigh number $\left(R=\alpha g d^{3} \Delta T / \nu \kappa\right)$ and the Marangoni number $(M=\gamma d \Delta T / \rho \nu \kappa)$ measure the relative importance of buoyancy and thermocapillarity forces. They are not independent but related by a constant $\Gamma=\gamma / \rho \alpha g d^{2}(M=\Gamma R)$. The limit $\Gamma \rightarrow 0$ corresponds to Rayleigh-Bénard convection while $\Gamma \rightarrow \infty$ is reached when gravity forces are absent or the thickness of the fluid is very small $\left(R \sim d^{2} M\right)$. In this work we will analyse three situations: a) $\Gamma=0.05$, corresponding to the experiments in refs. [10], [11], b) $\Gamma=1$, for some situations 
analysed in ref. [9] and c) $\Gamma=100$, when surface tension is much greater that buoyancy effects, like in microgravity. In order to determine completely the problem we must give also some boundary conditions (bc). The bottom is assumed to be rigid and conducting

$$
\mathbf{v}=0, T=T_{0} \quad \text { at } \quad z=0
$$

while for the upper surface, assumed to be nondeformable (which is a good approximation for layers which are thick enough) and partially conducting we consider heat transfer and the so called Marangoni condition:

$$
\frac{\partial u}{\partial z}+M \frac{\partial T}{\partial r}=\frac{\partial v}{\partial z}+\frac{M}{r} \frac{\partial T}{\partial \phi}=w=\frac{\partial T}{\partial z}+B i\left(T-T_{r e f}\right)=0 \quad \text { at } \quad z=1,
$$

where $T_{r e f}$ is a reference temperature, $(u, v, w)$ are the components of the velocity in cylindrical coordinates $(r, \phi, z)$ and $B i$ stands for the Biot number, a phenomenological parameter that accounts for the heat transfer between the fluid and the air. The limit $B i \rightarrow \infty$ corresponds to a perfect conducting medium above the fluid while $B i \rightarrow 0$ corresponds to an insulator material. We use for the rest of this paper the value $B i=0.1$ typical in experimental situations |16.

In a finite container we must consider the horizontal extend by means of another nondimensional parameter, the aspect ratio $a$, defined as the ratio between the radius and the height of the cylindrical container $(a=R / d)$. To complete the problem we need some bc's on the sidewall that depend on the geometry of the vessel. For example, adiabatic walls are described by the condition:

$$
\frac{\partial T}{\partial r}=0 \quad \text { at } \quad r=a .
$$

In usual experiments [9, 11] plastic sidewalls, matching that bc are used. For the velocity, instead of the usual non-slip condition we take non-deformable walls on which the tangential vorticity vanishes (slippery walls)

$$
u=\frac{\partial}{\partial r}(r v)=\frac{\partial w}{\partial r}=0 \quad \text { at } \quad r=a,
$$

This bc is used because the problem become separable, so analytical methods can be used. Slippery "walls" are obtained in the separation among convective cells in a big aspect ratio system. Although this b.c. does not correspond to that used in experiments, the corresponding solutions are expected to reproduce qualitatively the experimental facts. 
Once the equations governing the dynamics are given we can seek for solutions of these equations. In particular, we are interested in those solutions that appear when the trivial one (the conductive state) becomes unstable.

\section{Critical modes}

In this section we briefly recall the main results of the linear analysis of the equations and b.c. The conductive state has velocity and temperature fields in the form:

$$
\mathbf{v}_{\text {cond }}=0, T_{\text {cond }}=-z+T_{0}
$$

and conserving only the linear terms we obtain:

$$
\begin{array}{r}
\nabla \cdot \mathbf{v}=0 \\
\frac{\partial \mathbf{v}}{\partial t}=\operatorname{Pr}\left(-\nabla \pi+R \theta \mathbf{e}_{\mathbf{z}}+\nabla^{2} \mathbf{v}\right) \\
\frac{\partial \theta}{\partial t}=w+\nabla^{2} \theta
\end{array}
$$

with bc:

$$
\begin{aligned}
\frac{\partial u}{\partial z}+M \frac{\partial \theta}{\partial x}=\frac{\partial v}{\partial z}+M \frac{\partial \theta}{\partial y}=w=\frac{\partial \theta}{\partial z}+B i \theta=0 & \text { at } \quad & \text { at } \quad z=0 \\
\frac{\partial \theta}{\partial r}=u=\frac{\partial}{\partial r}(r v)=\frac{\partial w}{\partial r}=0 & \text { at } & r=a
\end{aligned}
$$

Solutions of these equations can be written as: $\mathbf{v}(\mathbf{r}, t)=\mathbf{v}(\mathbf{r}) e^{s t}, \theta(\mathbf{r}, t)=\theta(\mathbf{r}) e^{s t}, \pi(\mathbf{r}, t)=$ $\pi(\mathbf{r}) e^{s t}$, where $s$ is the growth rate. When marginal stability $(s=0)$ holds, we have:

$$
\begin{aligned}
\nabla \cdot \mathbf{v} & =0 . \\
-\nabla \pi+R \theta \mathbf{e}_{\mathbf{z}}+\nabla^{2} \mathbf{v} & =0 . \\
\nabla^{2} \theta+w & =0 .
\end{aligned}
$$

Separation of variables allows to consider solutions of the system (15)-(17) with bc (12)-(14) given by:

$$
\left.\begin{array}{l}
u_{m i j}(r, \phi, z)=\left(1 / k_{m i}\right) \cos \left(m \phi+\phi_{m}\right) J_{m}^{\prime}\left(k_{m i} r\right) D W_{m i j}(z) \\
v_{m i j}(r, \phi, z)=\left(-m / k_{m i}^{2} r\right) \sin \left(m \phi+\phi_{m}\right) J_{m}\left(k_{m i} r\right) D W_{m i j}(z) \\
w_{m i j}(r, \phi, z)=\cos \left(m \phi+\phi_{m}\right) J_{m}\left(k_{m i} r\right) W_{m i j}(z) \\
\theta_{m i j}(r, \phi, z)=\cos \left(m \phi+\phi_{m}\right) J_{m}\left(k_{m i} r\right) \Theta_{m i j}(z)
\end{array}\right\}
$$


where $m=0,1,2, \ldots$ is the azimuthal wavenumber, $J_{m}$ is the Bessel function of order $m$ and $i$ is the radial wavenumber, which indexes the values $k_{m i}$ satisfying the condition $J_{m}^{\prime}(k a)=0$. Due to symmetry there is no bc for the azimuthal equation, so the phase is not fixed and must be taken into account. This differs from the analysis of Rosenblat et al [四], in which the phase dynamics was not considered.

The functions $W_{m i j}(z), \Theta_{m i j}(z)$ are solutions of the system

$$
\begin{aligned}
& \left(D^{2}-k^{2}\right)^{2} W-R k^{2} \Theta=0 . \\
& \left(D^{2}-k^{2}\right) \Theta+W=0
\end{aligned}
$$

with bc

$$
\begin{aligned}
& W=D W=\Theta=0 \quad \text { at } \quad z=0 . \\
& W=D \Theta+B i \Theta=D^{2} W+M k^{2} \Theta=0 \quad \text { at } \quad z=1,
\end{aligned}
$$

where $D \equiv \frac{d}{d z}$. These equations have solutions for several values of $R$ denoted by $R_{j}(M, B i, m, i, a)$, so the Rayleigh number can be considered as the eigenvalue of the former system (and $j$ may be considered as a vertical wavenumber). The critical Rayleigh number $\left(R_{c}\right)$ is the minimum eigenvalue for which equations (19)-(22) have a solution. It corresponds to the onset of convection. Nevertheless we must notice that in a real system we do not fix $M$ but $\Gamma$. Therefore, in order to obtain the critical value $R_{c}$ we eliminate $M$ using the relation $M=\Gamma R$. Then, the critical Rayleigh number is:

$$
R_{c}=\min _{m i j} R_{j}(\Gamma, B i, a, m, i)
$$

Once $R_{c}$ determined the rest of the eigenvalues $R_{j}\left(M_{c}, B i, a, m, i\right)$ and the eigenfuntions $W_{m i j}$, $\Theta_{m i j}$ are calculated keeping the Marangoni number fixed $M_{c}=\Gamma R_{c}$. In Fig. 1 we plot $R_{c}$ versus $a$ for the different modes for $\Gamma=0.05$. For $\Gamma=100$ we recover the results quoted in ref. [1]. Experiments are in qualitative agreement with these results, but a quantitative comparison is not possible mainly due to the idealized bc considered in our analysis. It was noticed that modes with $m=3$ appear for $a=5-6$ in experiments, a factor 2 bigger than our calculations [11]. Another discrepancy is that the mode $m=0$ is observed in convective cells with $a<1$ [9] a fact that cannot be explained theoretically. However, numerical simulations of the Rayleigh-Benard problem with realistic lateral bc show the same sequence as in the present paper 17. 


\section{Weakly nonlinear expansion}

We perform a Galerkin-Eckhaus expansion of the fields in terms of eigenfunctions of the linear problem with time-depending amplitudes. After inserting this expansion in the PDE's and projecting over the modes of the adjoint problem, an infinite-dimensional system of ODE's for those amplitudes is obtained. Then, a center manifold reduction is performed into the few modes that remain dynamically active after all transients have relaxed.

Projecting the nonlinear system on the eigenfunctions of the adjoint system one arrives to (see Appendix)

$$
\left(1-\frac{R_{c}}{R}\right)\left\langle\theta_{m i j}^{*} w\right\rangle+\frac{1}{\Gamma^{2} R}\left(1-\frac{R_{m i j}^{*}}{R_{c}}\right)\left\langle w_{m i j}^{*} \theta\right\rangle=\left\langle\theta_{m i j}^{*} \frac{\partial \theta}{\partial t}\right\rangle+\left\langle\theta_{m i j}^{*}(\mathbf{v} \cdot \nabla) \theta\right\rangle,
$$

where $\mathbf{v}_{m i j}^{*}$ and $\theta_{m i j}^{*}$ are the adjoint modes and $\langle\cdots\rangle$ denotes the average over the fluid volume:

$$
\langle B\rangle \equiv \frac{1}{V} \int_{V} B d V=\frac{1}{\pi a^{2}} \int_{0}^{1} d z \int_{0}^{a} r d r \int_{0}^{2 \pi} d \phi B
$$

The velocity and temperature fields are expanded in terms of the modes of the linear problem:

$$
\left\{\begin{array}{c}
w \\
\theta
\end{array}\right\}=\sum_{m i j}\left(A_{m i j}(t) e^{i m \phi}+\bar{A}_{m i j}(t) e^{-i m \phi}\right) J_{m}\left(k_{m i} r\right)\left\{\begin{array}{c}
W_{m i j}(z) \\
\Theta_{m i j}(z)
\end{array}\right\}
$$

where $A_{m i j}=R_{m i j} e^{i \phi_{m}}$ is a complex amplitude with an indetermined phase $\phi_{m}$ included. We restrict our calculations to the neigbourhood of a codimension two (CT) point where two modes arise simultaneously. In our case the spatially homogeneous amplitude equations for these two modes match the normal form of such a CT point with $O(2)$ symmetry [12]:

$$
\begin{aligned}
& \dot{A}_{l}=p_{l} A_{l}+q_{l} \bar{A}_{l}^{m-1} A_{m}^{l} . \\
& \dot{A}_{m}=p_{m} A_{m}+q_{m} A_{l}^{m} \bar{A}_{m}^{l-1},
\end{aligned}
$$

where $p_{j}, q_{j}$ are functions of $\left|A_{l}\right|^{2},\left|A_{m}\right|^{2}$ and $A_{l}^{m} \bar{A}_{m}^{l}+\bar{A}_{l}^{m} A_{m}^{l}$ satisfying $p_{j}(0)=0, q_{j}(0)=0$. The last terms of these equations are called resonant terms. If $m+l-1 \leq 3$ these resonant terms appear in the normal form.

A strong resonance [14] is obtained between the two modes (11) and (21) for $a=a_{C T}$ (see Fig. 1), where the value $a_{C T}$ slightly depends on $\Gamma\left(a_{C T}=1.15\right.$ for $\Gamma=0.05$, and $a_{C T}=1.17$ for $\Gamma=1,100$ ) and the corresponding critical Rayleigh number for this point is denoted as $R_{C T}$ 
$\left(R_{C T}=530\right.$ for $\Gamma=0.05, R_{C T}=80.5$ for $\Gamma=1, R_{C T}=0.894$ for $\left.\Gamma=100\right)$. Under these conditions the normal form is:

$$
\begin{aligned}
& \dot{A}_{1}=\mu_{1} A_{1}+\alpha_{1} \bar{A}_{1} A_{2}-a_{1} A_{1}\left|A_{1}\right|^{2}-b_{1} A_{1}\left|A_{2}\right|^{2} . \\
& \dot{A}_{2}=\mu_{2} A_{2}-\alpha_{2} A_{1}^{2}-a_{2} A_{2}\left|A_{2}\right|^{2}-b_{2} A_{2}\left|A_{1}\right|^{2}
\end{aligned}
$$

where we have taken $p_{1}=\mu_{1}-a_{1}\left|A_{1}\right|^{2}-b_{1}\left|A_{2}\right|^{2}+\mathcal{O}\left(A^{3}\right), p_{2}=\mu_{2}-a_{2}\left|A_{2}\right|^{2}-b_{2}\left|A_{1}\right|^{2}+\mathcal{O}\left(A^{3}\right)$, $q_{1}=\alpha_{1}+\mathcal{O}\left(A^{2}\right), q_{2}=-\alpha_{2}+\mathcal{O}\left(A^{2}\right)$.

The form of Eqs. (29)-(30) does not depend on the details of the system, but arises from the resonance and the symmetry properties. The value of the coefficients depends on the specific problem and on the truncation order in the Galerkin-Eckhaus expansion. We consider the critical modes (11), (21) and a finite set of stable modes with lower growth rate, i.e., ((01), (31), (41), (12)), which are then eliminated using the center manifold reduction (see Appendix for details). The values of the coefficients under these approximations are given in Table 1.

Eqs. (29)-(30) have been studied by several authors [14, [15. Here we summarize the main results. Using polar coordinates for the amplitudes $A_{1}=r_{1} e^{i \phi_{1}}$ and $A_{2}=r_{2} e^{i \phi_{2}}$ system (29)-(30) is written

$$
\begin{aligned}
& \dot{r_{1}}=\alpha_{1} r_{1} r_{2} \cos \Phi+\mu_{1} r_{1}-a_{1} r_{1}^{3}-b_{1} r_{1} r_{2}^{2} \\
& \dot{r_{2}}=-\alpha_{2} r_{1}^{2} \cos \Phi+\mu_{2} r_{2}-a_{2} r_{2}^{3}-b_{2} r_{2} r_{1}^{2} \\
& \dot{\Phi}=\left(\alpha_{2} \frac{r_{1}^{2}}{r_{2}}-2 \alpha_{1} r_{2}\right) \sin \Phi
\end{aligned}
$$

with $\Phi=2 \phi_{1}-\phi_{2}$. (Rotational invariance allows to remove one of the phase equations).

There are three different types of steady-state solutions of Eqs. (31)-(33):

1) Pure modes $\left(P_{ \pm}\right)$:

$$
r_{1}=0, r_{2}^{2}=\frac{\mu_{2}}{a_{2}}, \Phi_{+}=0, \Phi_{-}=\pi
$$

These solutions represent convection with only one mode and correspond to a temperature field in the form (see Eq. (26)):

$$
\theta(r, \phi) \propto \sqrt{\frac{\mu_{2}}{a_{2}}} \cos (2 \phi) J_{2}\left(k_{21} r\right) \quad\left(P_{-}\right)
$$

For the sake of experimental comparison we plot the perturbation of the temperature field on the upper surface for the pure modes (21) and (11) in Fig. 2, although only the former one 
is solution of Eq. (34). The pure mode (21) has two reflection $\left(Z_{2}\right)$ symmetries and the (11) only the reflection respect to the diagonal that unites the upflow and downflow motions. In experiments shadowgraphy and schlieren techniques are used to analyse the patterns [10], [11]. In the corresponding images downflow motions appear as dark zones and upflow motions as bright zones. The mode (21) must give images with a cold line (downflow) in a diagonal that joins two black zones, while the mode (11) gives up and down motions near the sidewalls on the two opposite sites of a diagonal.

Pure mode solutions exist provided $a_{2} \mu_{2}>0$ and lose their stability to mixed modes when

$$
\mu_{1}>\frac{b_{1} \mu_{2}}{a_{2}} \pm \alpha_{1}\left(\frac{\mu_{2}}{a_{2}}\right)^{\frac{1}{2}}
$$

that we will denote line $\Pi$.

2) Mixed modes $\left(M_{ \pm}\right)$:

$$
\begin{aligned}
& 0=\mu_{1} \pm \alpha_{1} r_{2}-a_{1} r_{1}^{2}-b_{1} r_{2}{ }^{2}, \\
& 0=\mu_{2} r_{2} \mp \alpha_{2} r_{1}^{2}-b_{2} r_{1}^{2} r_{2}-a_{2} r_{2}^{3}, \\
& \Phi_{+}=0, \Phi_{-}=\pi .
\end{aligned}
$$

The two mixed modes represent mixed states of modes (11) and (21) (Eq. (26)):

$$
\theta(r, \phi) \propto r_{1} \cos (\phi) J_{1}\left(k_{11} r\right)+r_{2} \cos (2 \phi) J_{2}\left(k_{21} r\right)\left(M_{+}\right)
$$

Two examples are given in Fig. 3. Notice that these two modes mix in such a way that only one of the two $Z_{2}$ symmetries of the (21) mode is broken, i.e, the angle between these two modes is $0\left(M_{+}\right)$or $\pi\left(M_{-}\right)$. The mixed mode $M_{+}$undergoes a Hopf bifurcation on the line given by (37) together with

$$
a_{1} r_{1}^{2}+a_{2} r_{2}^{2}=\frac{\alpha_{2} r_{1}^{2}}{2 r_{2}}
$$

which gives rise to standing waves (SW) characterized by $\dot{\Phi}=0$, but time-dependent amplitudes $r_{1}$ and $r_{2}$. We will denote $\Lambda$ the line in which this ocurs.

3) Rotating waves (RW):

$$
\begin{aligned}
& r_{1}^{2}=2 \frac{\alpha_{1}}{\alpha_{2}} r_{2}^{2}, r_{2}^{2}=\frac{2 \mu_{1}+\mu_{2}}{\Delta}, \\
& \cos \Phi=\frac{1}{\alpha_{1} r_{2}} \frac{\mu_{2}\left[2\left(\alpha_{1} / \alpha_{2}\right) a_{1}+b_{1}\right]-\mu_{1}\left[2\left(\alpha_{1} / \alpha_{2}\right) b_{2}+a_{2}\right]}{\Delta}
\end{aligned}
$$


with

$$
\Delta \equiv 4 \frac{\alpha_{1}}{\alpha_{2}} a_{1}+2 b_{1}+2 \frac{\alpha_{1}}{\alpha_{2}} b_{2}+a_{2}
$$

At first order in perturbations they can be represented as:

$$
\theta(r, \phi) \propto \sqrt{\frac{2 \mu_{1}+\mu_{2}}{\Delta}}\left(\sqrt{2 \frac{\alpha_{1}}{\alpha_{2}}} \cos \left(\phi+\dot{\phi}_{1} t\right) J_{1}\left(k_{11} r\right)+\cos \left(2\left(\phi+\dot{\phi}_{1} t\right)-\Phi\right) J_{2}\left(k_{21} r\right)\right)
$$

with $\Delta, \dot{\phi}_{1}$ and $\Phi$ given by Eqs. (42), (45) and (41).

In this state the phases of the two modes $\phi_{1}$ and $\phi_{2}$ are variable but the relative phase $\Phi$ is constant and different from zero. So, the remaining $Z_{2}$ symmetry of the (11) mode is broken, as it is shown in the two examples given in Fig. 4. These rotating waves exist provided that $\left(2 \mu_{1}+\mu_{2}\right) \Delta>0$ and $|\cos \Phi| \leq 1$, i.e.,

$$
\left[\left(2 \frac{\alpha_{1}}{\alpha_{2}} a_{1}+b_{1}\right) \mu_{2}-\left(2 \frac{\alpha_{1}}{\alpha_{2}} b_{2}+a_{2}\right) \mu_{1}\right]^{2} \leq \alpha_{1}^{2}\left(2 \mu_{1}+\mu_{2}\right) \Delta
$$

and bifurcate off $M_{ \pm}$at $|\cos \Phi|=1$ (line $\Omega$ ).

The pattern of these RW rotates with a phase velocity

$$
\dot{\phi}_{2}=2 \dot{\phi}_{1}=-2 \alpha_{1} r_{2} \sin \Phi=-2 \alpha_{1} \sqrt{\frac{2 \mu_{1}+\mu_{2}}{\Delta}\left(1-\frac{\left(\beta \mu_{2}-\gamma \mu_{1}\right)^{2}}{\alpha_{1}^{2} \Delta\left(2 \mu_{1}+\mu_{2}\right)}\right)},
$$

where $\beta \equiv 2\left(\alpha_{1} / \alpha_{2}\right) a_{1}+b_{1}$ and $\gamma \equiv a_{2}+2\left(\alpha_{1} / \alpha_{2}\right) b_{2}$. In Fig. 5 the phase velocity (45) is plotted as a function of $R$ for several values of the aspect ratio $a$. This has been calculated from the equation

$$
\mu_{m}=\frac{1}{\tau_{m}}\left\langle\theta_{m 11}^{*} w_{m 11}\right\rangle\left(1-\frac{R_{c_{m}}(a)}{R}\right), m=1,2,
$$

that relates the control parameters $\mu_{1}, \mu_{2}$ with the physically relevant nondimensional numbers $R$ and $a$.

The RW can become unstable to modulated waves (MW) with time dependent amplitudes and phase velocities $\dot{\phi}_{1}, \dot{\phi}_{2} \neq 0$.

\subsection{Heteroclinic orbit}

When condition

$$
0>\mu_{1}-\frac{b_{1} \mu_{2}}{a_{2}}>-\alpha_{1}\left(\frac{\mu_{2}}{a_{2}}\right)^{\frac{1}{2}}
$$


is fulfilled, a stable heteroclinic orbit exists. We gathered in Fig. 6a the form of the patterns that may appear in four representative points of that orbit. Usually a point of this orbit corresponds to a mixture of the modes (21) and (11), but when it reaches the $r_{1}$-axis the phase suddenly jumps in $\pi$. Therefore, the amplitude of the mode (21) reverses sign. When $r_{1} \simeq 0$ there is a pure mode (21) that varies its phase in $\pi / 2$. Experimentally this heteroclinic orbit would lead to patterns evolving from one pure mode to another with a phase changed in $\pi / 2$. This orbit is structurally stable to changes in the parameters $\alpha_{i}, a_{i}, b_{i}$. But it is interesting to notice that slight changes in the form of Eqs. (29)-(30) (imperfect symmetry) destroy the heteroclinic orbit that degenerates into a limit cycle. In fact numerical noise suffices to convert the heteroclinic orbit into a periodic orbit in numerical simulations of Eqs.(29)-(30) shown in Fig. 6b. Small thermal noise, cell imperfections, etc, unavoidable in real experiments will prevent the formation of the heteroclinic orbit which is replaced by periodic motions between modes (11) and (21).

In Fig. 7 the bifurcation diagram is plotted as function of $\mu_{1}, \mu_{2}$ for $\Gamma=0.05$. This diagram can be plotted in the plane $(R, a)$ as shown in Fig. 8. In Fig. 9 the stability diagrams for $\Gamma=1$ and $\Gamma=100$ are represented. The quotations in these figures correspond to the stability regions of the different solutions. From Figs. 7 and 8 it can be deduced that for $a>a_{C T}$ the dynamics begins with a mixed mode $\left(M_{-}\right)$that changes into a rotating wave $(R W)$ when the heating is increased. For $a<a_{C T}$ convection starts in a pure mode $(P)$ that bifurcates to a mixed mode $\left(M_{+}\right)$. This is replaced by a heteroclinic orbit $(H)$ when the control parameter is rised. Notice that $(H)$ is stable in a full region of parameter space. Therefore we hope that a periodic dynamics that arises from this heteroclinic connection would be reachable in experiments with suitable conditions.

\section{Conclusions}

We performed an analysis of BM convection in a cylindrical container with small aspect ratio. For the linear problem we calculated the marginal curve $R_{c}(a)$ and the unstable modes as a function of the aspect ratio $a$ for several values of the ratio between surface tension and buoyancy effects $(\Gamma)$. These results have been obtained for idealized lateral boundary conditions (slippery walls). Therefore, they allow only for qualitative comparison with experiments. The sequence of modes and the critical Rayleigh number compares quite well with experimental findings. 
However, the critical aspect ratios calculated are smaller than those observed in experiments. This has also been obtained in the case of BM convection in a square container 18. A possible explanation is that the viscous boundary layer in real non-slippery walls rises the aspect ratio respect to an idealized slippery one.

The nonlinear analysis is performed near a codimension two (CT) point where two stationary pure modes are simultaneously unstable. We choose a situation where a resonance 2:1 between modes (11) and (21) appears. A center manifold reduction is done to determine the coefficients of the normal form in the neighbourhood of that point. Depending on the parameter values the stationary solutions are: a) a pure mode (P) (with two reflection symmetries), mixed modes (M) (with only one $Z_{2}$ symmetry), rotating waves (RW) (without reflection symmetry) and a heteroclinic orbit $(\mathrm{H})$. We calculate the dependence of rotation velocity of RW as a function of the order parameter. The heteroclinic orbit is quite robust and appears as a stable solution in a big region of the parameter space (see Figs. 8, 9). In an experiment this orbit will degenerate into a periodic alternancy between two modes that could be detected in a wide region in the parameter space. We hope that new experiments will confirm the presence of a heteroclinic conection and of rotating waves in this system.

\section{Acknowledgements}

We acknowledge fruitful comments and discussions with P. Dauby and G. Lebon (Liege, Belgium), G. Mindlin (Buenos Aires, Argentina), H. Mancini, A. Garcimartín and D. Maza (Pamplona). This work has been partilly funded by PIUNA (Universidad de Navarra) and by the DGICYT (Spanish Government) under grant PB94-0708, and with an European Union

contract ERBCHRXT940546. B.E. acknowledges the Basque Government for a fellowship (BFI95.035) and D.K. the kind hospitality of the Universidad de Navarra. 


\section{Appendix}

The adjoint linear problem is given by the equations

$$
\begin{aligned}
& \nabla^{2} \mathbf{v}^{*}-\nabla \pi^{*}+\theta^{*} \mathbf{e}_{z}=\mathbf{0} . \\
& \nabla \cdot \mathbf{v}^{*}=0 \\
& \nabla^{2} \theta^{*}+R^{*} w^{*}=0
\end{aligned}
$$

with bc

$$
\begin{aligned}
& u^{*}=v^{*}=w^{*}=\theta^{*}=0, \quad \text { at } \quad z=0, \\
& w^{*}=\frac{\partial u^{*}}{\partial z}=\frac{\partial v^{*}}{\partial z}=\frac{\partial \theta^{*}}{\partial z}+B i \theta^{*}+M \frac{\partial w^{*}}{\partial z}=0, \quad \text { at } \quad z=1, \\
& u^{*}=\frac{\partial}{\partial r}\left(r v^{*}\right)=\frac{\partial w^{*}}{\partial r}=0, \quad \text { at } \quad r=a .
\end{aligned}
$$

This problem is separable and the eigenvalues $R_{m i j}^{*}$ are the same of those of the system (15)-(17). The eigenfunctions are:

$$
\begin{aligned}
& u_{m i j}^{*}=k_{m i} \cos (m \phi) J_{m}^{\prime}\left(k_{m i} r\right) D W_{m i j}^{*}(z) \\
& v_{m i j}^{*}=-(m / r) \sin (m \phi) J_{m}\left(k_{m i} r\right) D W_{m i j}^{*}(z) \\
& w_{m i j}^{*}=k_{m i j}^{2} \cos (m \phi) J_{m}\left(k_{m i} r\right) W_{m i j}^{*}(z) \\
& \theta_{m i j}^{*}=\cos (m \phi) J_{m}\left(k_{m i} r\right) \Theta_{m i j}^{*}(z)
\end{aligned}
$$

with $W_{m i j}^{*}$ and $\Theta_{m i j}^{*}$ solutions of the system

$$
\begin{aligned}
& \left(D^{2}-k^{2}\right) \Theta^{*}+R k^{2} W^{*}=0 \\
& \left(D^{2}-k^{2}\right)^{2} W^{*}-\Theta^{*}=0
\end{aligned}
$$

with bc

$$
\begin{aligned}
& \Theta^{*}=W^{*}=D W^{*}=0, \quad \text { at } \quad z=0 . \\
& W^{*}=D^{2} W^{*}=D \Theta^{*}+B i \Theta^{*}+k^{2} M_{c} D W^{*}=0, \quad \text { at } \quad z=1 .
\end{aligned}
$$

These eigenfunctions are introduced to obtain

$$
\begin{aligned}
(1 & \left.-\frac{R_{c}}{R}\right)\left\langle\theta_{m i j}^{*} w\right\rangle+\frac{1}{\Gamma^{2} R}\left(1-\frac{R_{m i j}^{*}}{R_{c}}\right)\left\langle w_{m i j}^{*} \theta\right\rangle \\
& =\left\langle\theta_{m i j}^{*} \frac{\partial \theta}{\partial t}+\frac{1}{P r} \mathbf{v}_{m i j}^{*} \cdot \frac{\partial \mathbf{v}}{\partial t}\right\rangle+\left\langle\theta_{m i j}^{*}(\mathbf{v} \cdot \nabla) \theta\right\rangle+\frac{1}{P r}\left\langle\mathbf{v}_{m i j}^{*} \cdot(\mathbf{v} \cdot \nabla) \mathbf{v}\right\rangle
\end{aligned}
$$


that in the limit $\operatorname{Pr} \rightarrow \infty$ leads to

$$
\left(1-\frac{R_{c}}{R}\right)\left\langle\theta_{m i j}^{*} w\right\rangle+\frac{1}{\Gamma^{2} R}\left(1-\frac{R_{m i j}^{*}}{R_{c}}\right)\left\langle w_{m i j}^{*} \theta\right\rangle=\left\langle\theta_{m i j}^{*} \frac{\partial \theta}{\partial t}\right\rangle+\left\langle\theta_{m i j}^{*}(\mathbf{v} \cdot \nabla) \theta\right\rangle .
$$

Introducing expression (26) for the temperature and velocity fields, we obtain an infinite set of ODE's for the amplitudes. In order to perform a center manifold reduction we must take into account the critical modes and the set of stable modes that appear as cuadratical interaction of these ones (so they give thirth order terms in the center manifold equations). This is, the set: $(0 i),(1 j),(2 j),(3 i),(4 i), i=1,2, \ldots, j=2,3, \ldots$. Since this is again an infinite set we will retain only those modes with the smallest eigenvalues $R_{m i j}^{*}$ (see Table 2). Moreover we have checked that only modes $(01),(31),(41),(12)$ give an estimable contribution to the coefficients of the normal form. With this, the following amplitude equations are obtained:

$$
\left.\begin{array}{rl}
\tau_{01} \dot{A}_{01}= & \tilde{\epsilon}_{01} A_{01}+\alpha_{010101}\left(A_{01}^{2}+\left|A_{01}\right|^{2}\right)+\frac{\alpha_{011111}}{2}\left|A_{11}\right|^{2}+\frac{\alpha_{011212}}{2}\left|A_{12}\right|^{2}+\frac{\alpha_{012121}}{2}\left|A_{21}\right|^{2} \\
& +\frac{\alpha_{013131}}{2}\left|A_{31}\right|^{2}+\frac{\alpha_{014141}}{2}\left|A_{41}\right|^{2} . \\
\tau_{11} \dot{A}_{11}= & \tilde{\epsilon}_{11} A_{11}+\alpha_{111121} \bar{A}_{11} A_{21}+\alpha_{111221} \bar{A}_{12} A_{21}+\alpha_{111101}\left(A_{11} A_{01}+A_{11} \bar{A}_{01}\right) \\
& +\alpha_{111201}\left(A_{12} A_{01}+A_{12} \bar{A}_{01}\right)+\alpha_{112131} \bar{A}_{21} A_{31}+\alpha_{113141} \bar{A}_{31} A_{41} . \\
\tau_{21} \dot{A}_{21}= & \tilde{\epsilon}_{21} A_{21}+\alpha_{211111} A_{11}^{2}+\alpha_{211212} A_{12}^{2}+\alpha_{211131} \bar{A}_{11} A_{31}+\alpha_{211231} \bar{A}_{12} A_{31} \\
& +\alpha_{212101}\left(A_{21} A_{01}+A_{21} \bar{A}_{01}\right)+\alpha_{212141} \bar{A}_{21} A_{41} \cdot \\
\tau_{31} \dot{A}_{31}= & \tilde{\epsilon}_{31} A_{31}+\alpha_{311121} A_{11} A_{21}+\alpha_{311221} A_{12} A_{21}+\alpha_{311141} \bar{A}_{11} A_{41}+\alpha_{311241} \bar{A}_{12} A_{41} \\
& +\alpha_{313101}\left(A_{31} A_{01}+A_{31} \bar{A}_{01}\right) . \\
\tau_{41} \dot{A}_{41}= & \tilde{\epsilon}_{41} A_{41}+\alpha_{414101}\left(A_{41} A_{01}+A_{41} \bar{A}_{01}\right)+\alpha_{411131} A_{11} A_{31}+\alpha_{411231} A_{12} A_{31} \\
& +\alpha_{412121} A_{21}^{2} . \\
\tau_{12} \dot{A}_{12}= & \tilde{\epsilon}_{12} A_{12}+\alpha_{121121} \bar{A}_{11} A_{21}+\alpha_{121221} \bar{A}_{12} A_{21}+\alpha_{121101}\left(A_{11} A_{01}+A_{11} \bar{A}_{01}\right) \\
& +\alpha_{121201}\left(A_{12} A_{01}+A_{12} \bar{A}_{01}\right)+\alpha_{122131} \bar{A}_{21} A_{31}+\alpha_{123141} \bar{A}_{31} A_{41} .
\end{array}\right\}
$$

with

$$
\begin{aligned}
& \tau_{m n}=\left\langle\theta_{m n 1}^{*} \theta_{m n 1}\right\rangle, m=1 \ldots 4, n=1,2, \\
& \tilde{\epsilon}_{i 1}=\left\langle\theta_{i 11}^{*} w_{i 11}\right\rangle\left(1-\frac{R_{c_{i}}}{R}\right) \equiv\left\langle\theta_{i 11}^{*} w_{i 11}\right\rangle \epsilon_{i 1}, i=1,2 \quad\left(\epsilon_{i} \equiv 1-\frac{R_{c_{i}}}{R}\right), \\
& \tilde{\epsilon}_{j k}=\left\langle\theta_{j k 1}^{*} w_{j k 1}\right\rangle\left(1-\frac{R_{c}}{R}\right)+\frac{\left\langle w_{j k 1}^{*} \theta_{j k 1}\right\rangle}{\Gamma^{2} R}\left(1-\frac{R_{j k 1}^{*}}{R_{c}}\right) \simeq \frac{\left\langle w_{j 11}^{*} \theta_{j 11}\right\rangle}{\Gamma^{2} R_{c}}\left(1-\frac{R_{j 11}^{*}}{R_{c}}\right), \\
& \text { if } R \simeq R_{c}(j=0,3,4, k=1 \text { or } j=1, k=2), \\
& \alpha_{i j k l m n}=\left\langle\theta_{i j 1}^{*}\left(\mathbf{v}_{k l 1} \cdot \nabla\right) \theta_{m n 1}\right\rangle
\end{aligned}
$$

After eliminating the modes $(01),(31),(41),(12)$ with a center manifold reduction, we obtain 
the equations for the critical modes (11), (21):

$$
\begin{aligned}
\tau_{11} \dot{A}_{11}= & \tilde{\epsilon}_{11} A_{11}+\alpha_{111121} A_{21} \bar{A}_{11}-\frac{\alpha_{111101} \alpha_{011111}}{\tilde{\epsilon}_{01}} A_{11}\left|A_{11}\right|^{2}-\left(\frac{\alpha_{110111} \alpha_{012121}}{\tilde{\epsilon}_{01}}\right. \\
& \left.+\frac{\alpha_{112131} \alpha_{311121}}{\tilde{\epsilon}_{31}}+\frac{\alpha_{111221} \alpha_{121121}}{\tilde{\epsilon}_{12}}\right) A_{11}\left|A_{21}\right|^{2} . \\
\tau_{21} \dot{A}_{21}= & \tilde{\epsilon}_{21} A_{21}+\alpha_{211111} A_{11}^{2}-\left(\frac{\alpha_{212101} \alpha_{012121}}{\tilde{\epsilon}_{01}}+\frac{\alpha_{212141} \alpha_{412121}}{\tilde{\epsilon}_{41}}\right) A_{21}\left|A_{21}\right|^{2} \\
& -\left(\frac{\alpha_{211131} \alpha_{311121}}{\tilde{\epsilon}_{31}}+\frac{\alpha_{212101} \alpha_{01111}}{\tilde{\epsilon}_{01}}+\frac{\alpha_{211112} \alpha_{121121}}{\tilde{\epsilon}_{12}}\right) A_{21}\left|A_{11}\right|^{2} .
\end{aligned}
$$

In Table 3 the values of these coefficients are given for $\Gamma=0.05$. Dividing these by $\tau_{11}$ and $\tau_{21}$ respectively, we obtain the coefficients given in Table 1 . 


\section{References}

[1] H. Bénard, Rev. Gén. Sci. Pure Appl. 11 (1900)

[2] M.C. Cross and P. C. Hohenberg, Rev. Mod. Phys. 65 (1993) 851.

[3] J.R.A. Pearson, J. Fluid Mech. 4 (1958) 489.

[4] S. Rosenblat, S.H. Davis and G.M. Homsy, J. Fluid Mech., 120 (1982) 91.

[5] S. Rosenblat, G.M. Homsy and S.H. Davis, J. Fluid Mech., 120 (1982) 123.

[6] L.E. Koschmieder, Bénard Cells and Taylor Vortices (Cambridge Univ. Press, Cambridge, 1994).

[7] P. Cerisier, R. Occelli, C. Pérez-García and C. Jamond, J. Physique 48 (1987) 569.

[8] M.F. Schatz, S.J. VanHook, W.D. McCormick, J.B. Swift and H.L. Swinney, , Phys. Rev. Lett. 75, (1995) 1938.

[9] E.L. Koschmieder and Prahl, J. Fluid Mech., 215 (1990) 571.

[10] G. Mindlin, T. Ondarcuhu, H.L. Mancini, C. Pérez-García and A. Garcimartín, Int. J. Bifur. Chaos, 4 (1994) 1121.

[11] T. Ondarcuhu. J. Millán, H.L. Mancini, A. Garcimartín and C. Pérez-García, Phys. Rev. E, 48 (1993) 1051.

[12] M. Golubitsky, I. Stewart and D.G. Schaeffer, Singularities and Groups in Bifurcation Theory II (Springer, New York, 1988).

[13] R. Friedrich and H. Haken, Phys. Rev. A 34 (1986) 2100

[14] G. Dangelmayr, Dyn. Stab. Syst. 1 (1986) 159.

[15] C.A. Jones and M.R.E. Proctor, Phys. Lett. A 121 (1987) 224; M.R.E. Proctor and C.A. Jones, J. Fluid Mech. 188 (1988) 301.

[16] H.L. Mancini, Doctoral Thesis, Universidad de Navarra (1994) (unpublished) 
[17] G.Z. Gershuni and E.M. Zhukhovitskii, Convective Stability of Incompressible Fluids (Keter, Jerusalem, 1976).

[18] P.C. Dauby and G. Lebon, Q. J. Mech. Appl. Math. 46 (1993) 483 


\section{Tables}

\begin{tabular}{|c|c||c|c||c|c|}
\hline \multicolumn{2}{|c||}{$\Gamma=0.05$} & \multicolumn{2}{c||}{$\Gamma=1$} & \multicolumn{2}{c|}{$\Gamma=100$} \\
\hline$\mu_{1}=.1947 \epsilon_{1}$ & $\mu_{2}=.3599 \epsilon_{2}$ & $\mu_{1}=0.06654 \epsilon_{1}$ & $\mu_{2}=0.09771 \epsilon_{2}$ & $\mu_{1}=0.05381 \epsilon_{1}$ & $\mu_{2}=0.09708 \epsilon_{2}$ \\
\hline$\alpha_{1}=.8376$ & $\alpha_{2}=.4069$ & $\alpha_{1}=0.1842$ & $\alpha_{2}=0.04952$ & $\alpha_{1}=0.03681$ & $\alpha_{2}=0.002350$ \\
\hline$a_{1}=17.45$ & $a_{2}=6.637$ & $a_{1}=2.130$ & $a_{2}=1.141$ & $a_{1}=0.006209$ & $a_{2}=0.003554$ \\
\hline$b_{1}=10.16$ & $b_{2}=28.36$ & $b_{1}=1.603$ & $b_{2}=4.655$ & $b_{1}=0.004855$ & $b_{2}=0.01384$ \\
\hline
\end{tabular}

Table 1: Coefficients of the normal form for several values of $\Gamma$.

\begin{tabular}{|c|c|c|c|}
\hline$R_{111}^{*}=530.3$ & $R_{211}^{*}=530.3$ & $R_{011}^{*}=696.4$ & $R_{311}^{*}=812.2$ \\
\hline$R_{411}^{*}=1326$ & $R_{121}^{*}=1332$ & $R_{221}^{*}=2403$ & $R_{321}^{*}=4039$ \\
\hline
\end{tabular}

Table 2: Eigenvalues of the adjoint problem.

\begin{tabular}{|c|c|c|c|c|}
\hline$\tilde{\epsilon}_{01}=-3.102$ & $\tilde{\epsilon}_{11}=1.592 \epsilon_{11}$ & $\tilde{\epsilon}_{21}=1.473 \epsilon_{21}$ & $\tilde{\epsilon}_{31}=-.4902$ & $\tilde{\epsilon}_{41}=-1.168$ \\
\hline$\tilde{\epsilon}_{12}=-3.889$ & $\tau_{11}=8.179$ & $\tau_{21}=4.093$ & $\alpha_{012121}=7.750$ & $\alpha_{011111}=34.68$ \\
\hline$\alpha_{111101}=-12.77$ & $\alpha_{112131}=9.211$ & $\alpha_{112111}=6.851$ & $\alpha_{211111}=-1.666$ & $\alpha_{211131}=4.255$ \\
\hline$\alpha_{212101}=-8.267$ & $\alpha_{311121}=-2.726$ & $\alpha_{412121}=-1.081$ & $\alpha_{212141}=7.040$ & $\alpha_{211112}=-7.594$ \\
\hline$\alpha_{111121}=6.851$ & $\alpha_{111221}=-11.545$ & $\alpha_{121121}=5.685$ & & \\
\hline
\end{tabular}

Table 3: Coefficients used in center manifold reduction for $\Gamma=0.05$. 


\section{Figure Captions.}

Figure 1: Marginal curve representing the critical Rayleigh number $R_{c}$ as a function of the aspect ratio $a$. The indexes $(m i)$ stand for the azimuthal $(\mathrm{m})$ and radial (i) wavenumbers. Only the modes with vertical wavenumber $j=1$ are represented because we have assumed the ordering $R_{m i 1}<R_{m i 2} \ldots$

Figure 2: Pure modes a) (11) and b) (21). Only the second one appears as a solution of Eq. 34. (Point a in Fig. 8).

Figure 3: Mixed mode a) $M_{+}$, b) $M_{-}$. (Points b and c in Fig. 8).

Figure 4: Rotating waves for a) $\Phi=2.0$ and b) $\Phi=2.25$. (Points d and e in Fig. 8).

Figure 5: Phase velocity $\dot{\phi}_{2}$ (in absolute value) as a function of Rayleigh number $R$ when a) $a=1.00$, b) $a=1.15$, c) $a=1.30$. The solid line corresponds to the case when the $R W$ are stable and the doted line when they are unstable. These $R W$ appear as a supercritical bifurcation. From Eqs. (45) and (46) it can be seen that, for $\epsilon \ll 1, \dot{\phi}_{2} \propto \epsilon^{\frac{1}{2}}$. $\left(\epsilon \equiv \frac{R-R_{c}}{R_{c}}\right)$.

Figure 6: a) Heteroclinic orbit. b) Temporal series of the amplitude $r_{1}$ when the system is in the former orbit. After a transient, the heteroclinic conexion merges into a limit cycle due to the numerical noise.

Figure 7: Bifurcation diagram in the plane $\mu_{1}, \mu_{2}$. Parentheses indicate unstable solutions. The lines $\Sigma_{1}$ and $\Sigma_{2}$ represent the limits of existence of the heteroclinic orbit, as given by Eq. (47). (For further explanations see the text).

Figure 8: Stability diagrams in the plane $(R, a)$. Only the stable solutions are represented. When more than one solution is stable, that with the smallest basin of attraction is represented in parenthesis. The points a, b, c, d, e, f correspond to the solutions shown in Figs. 2b, 3a, 3b, $4 \mathrm{a}, 4 \mathrm{~b}$ and 6 , respectively.

Figure 9: Stability diagram in $(R, a)$ space, for a) $\Gamma=1$ and b) $\Gamma=100$. The stability region for the heteroclinic orbit $(H)$ increses as $\Gamma$ increases. 


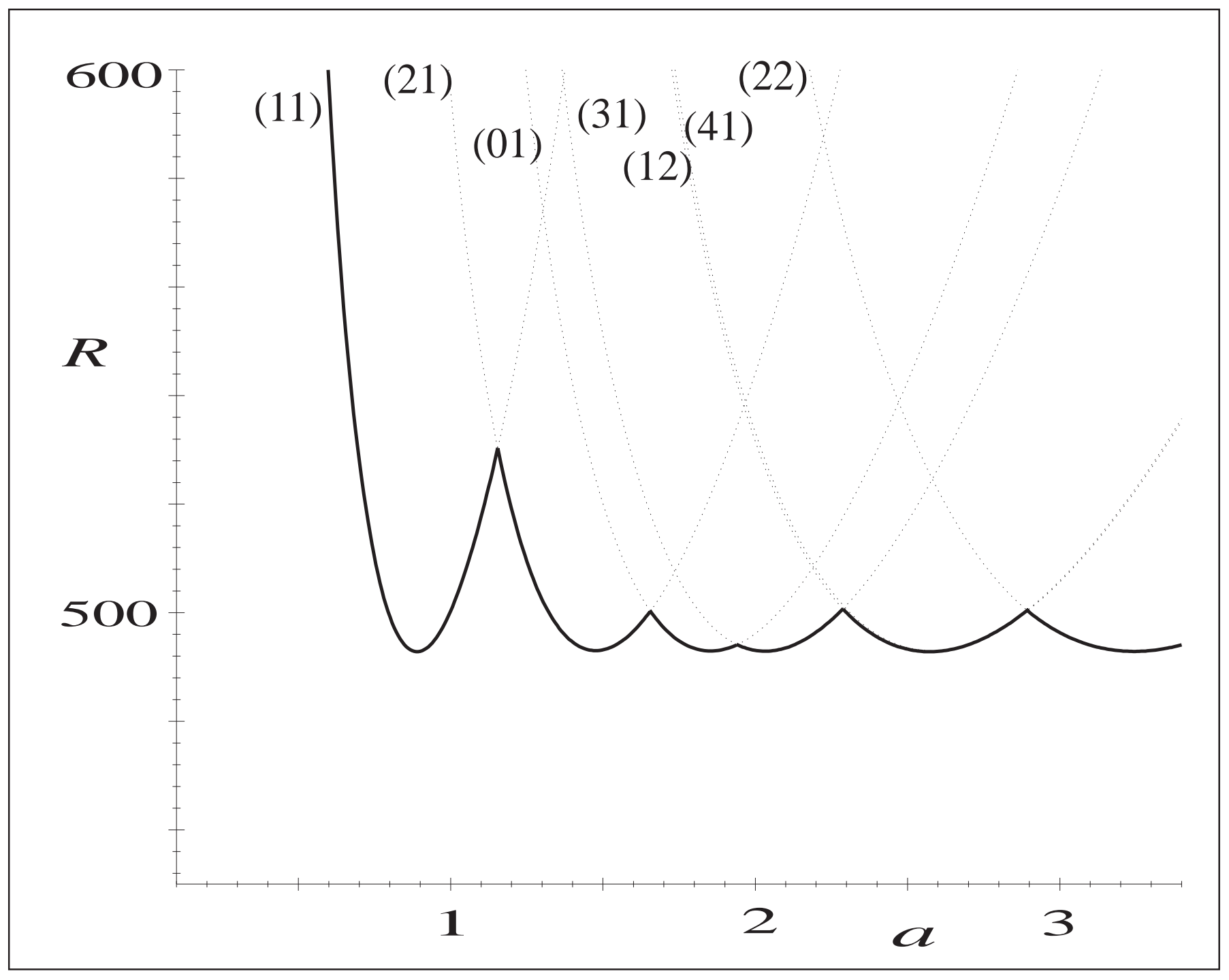

Figure 1. 


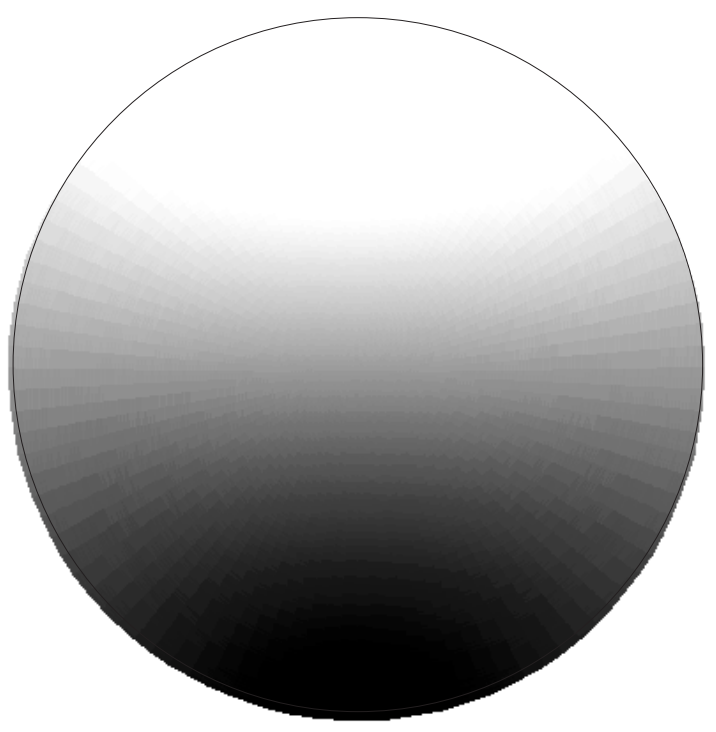

Figure 2a. 


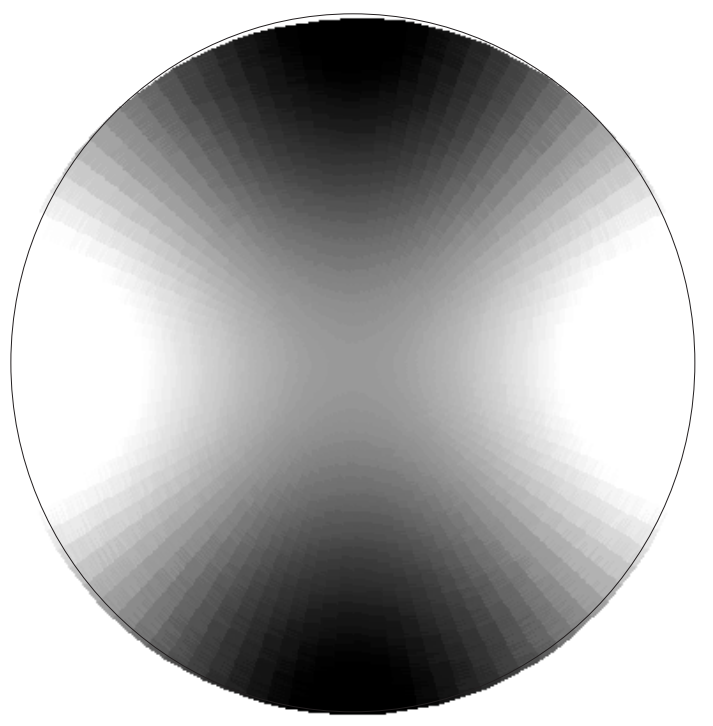

Figure $2 b$. 


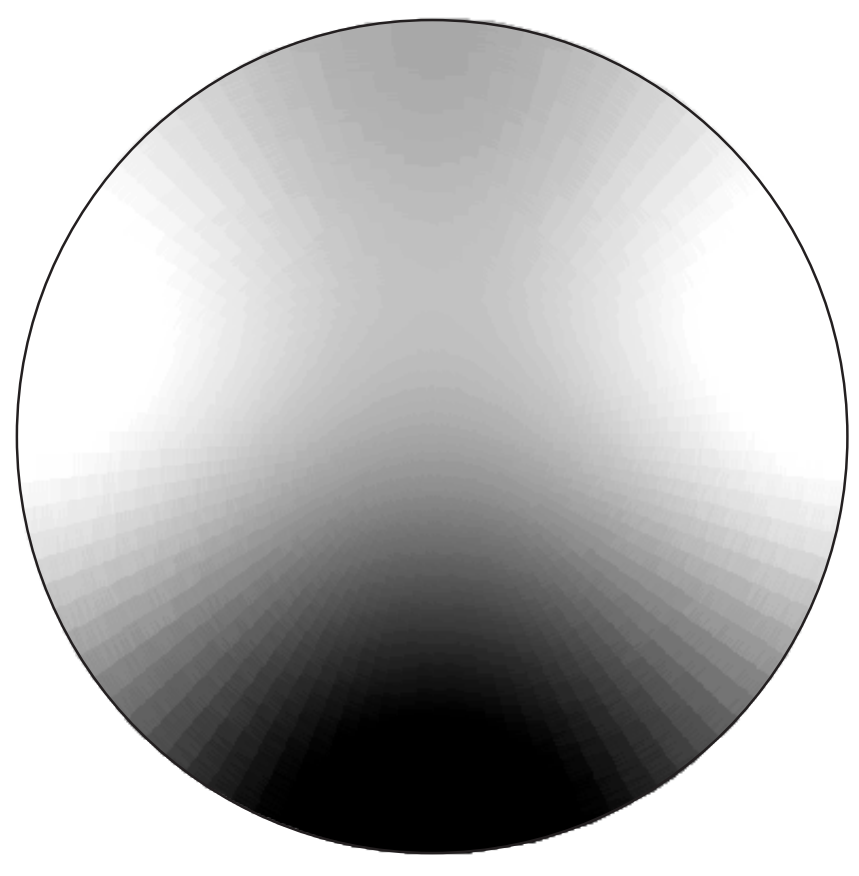

Figure 3a. 


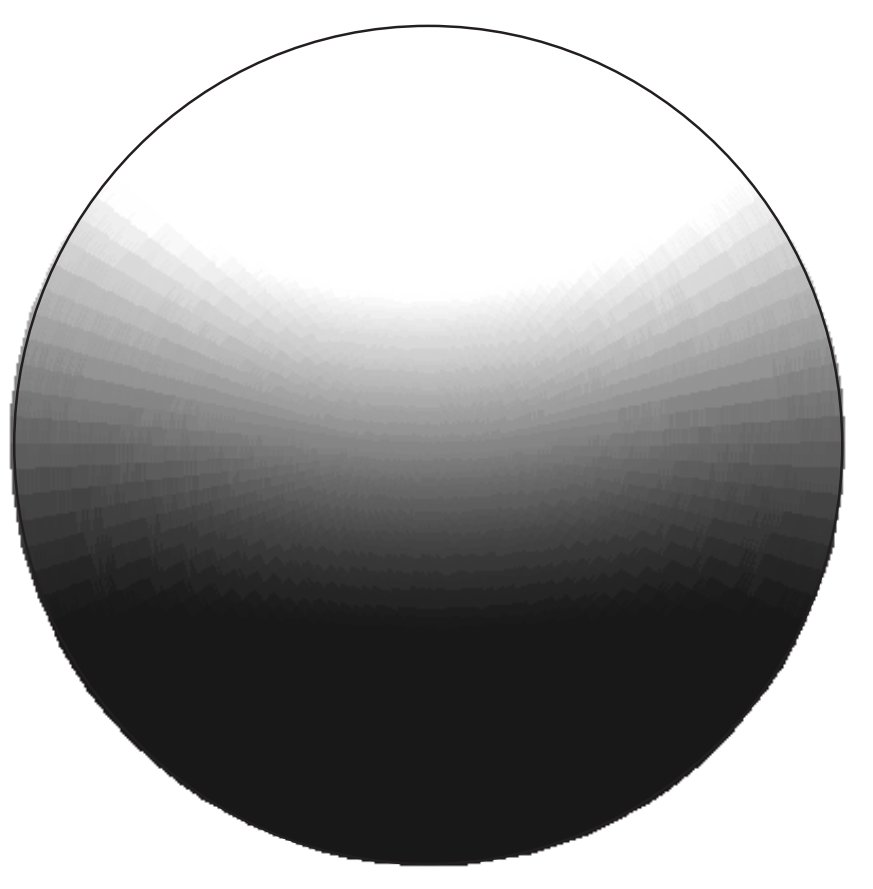

Figure $3 b$. 


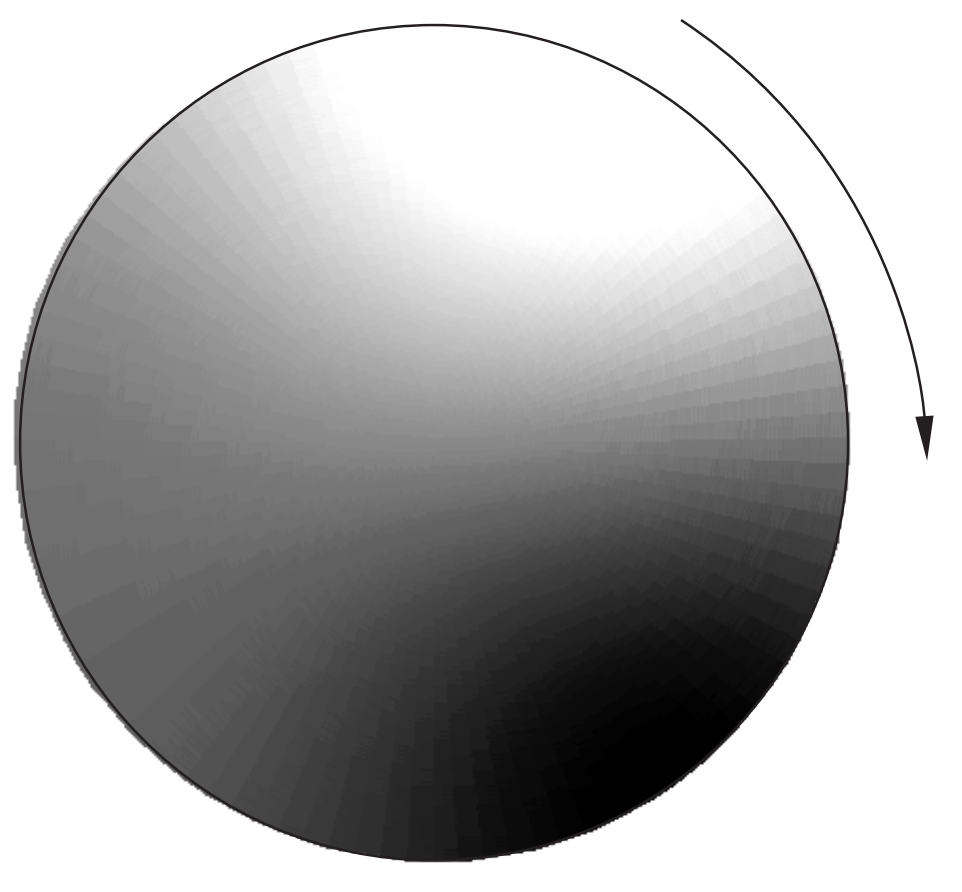

Figure 4a. 


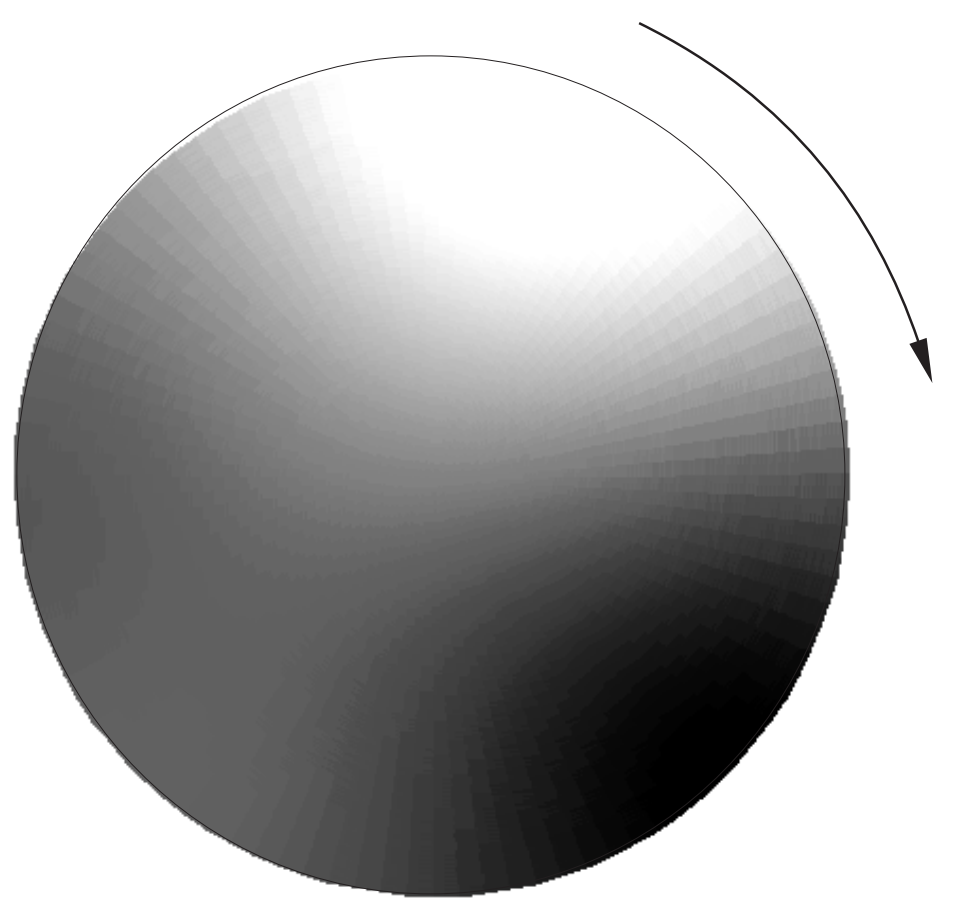

Figure 4b. 


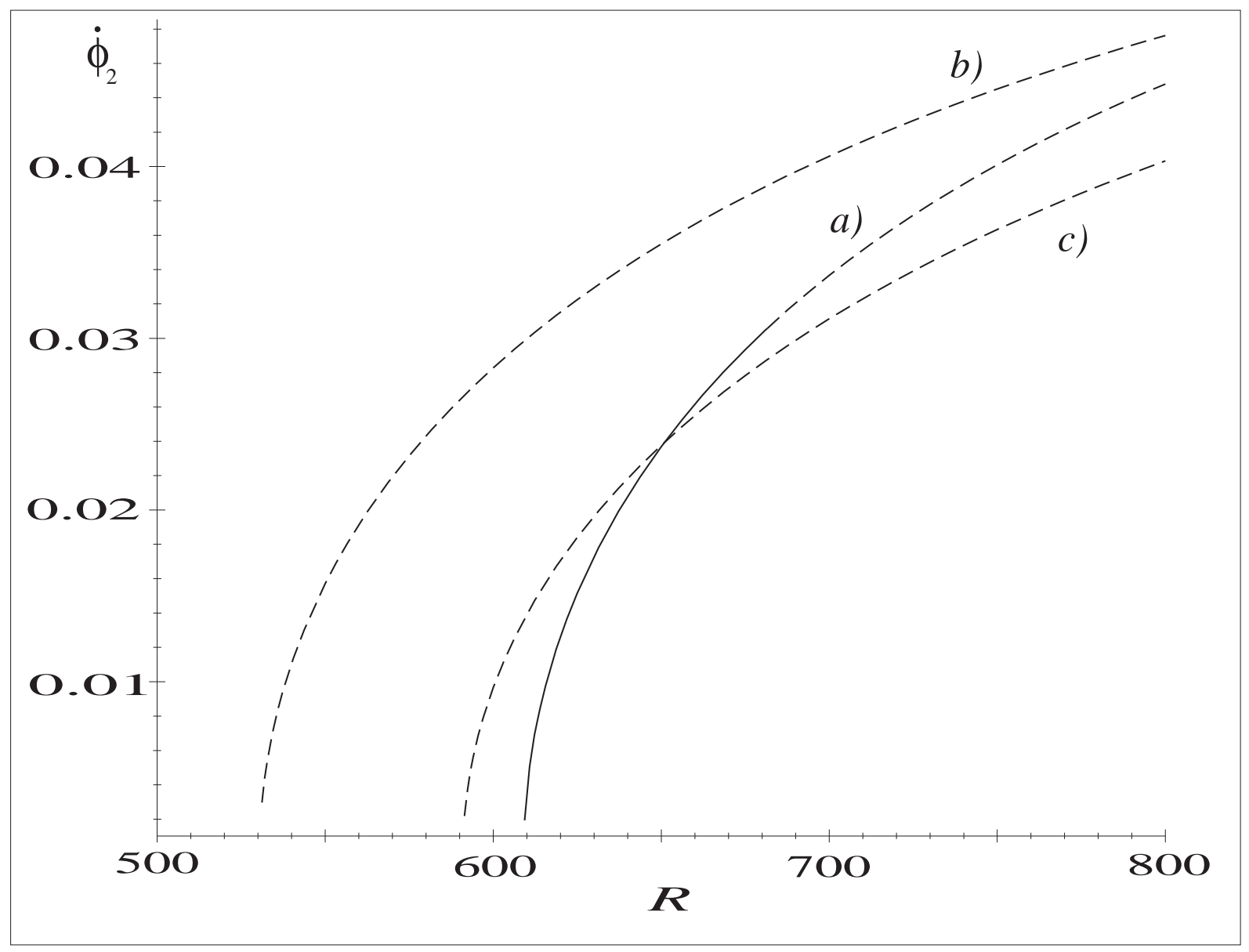

Figure 5. 


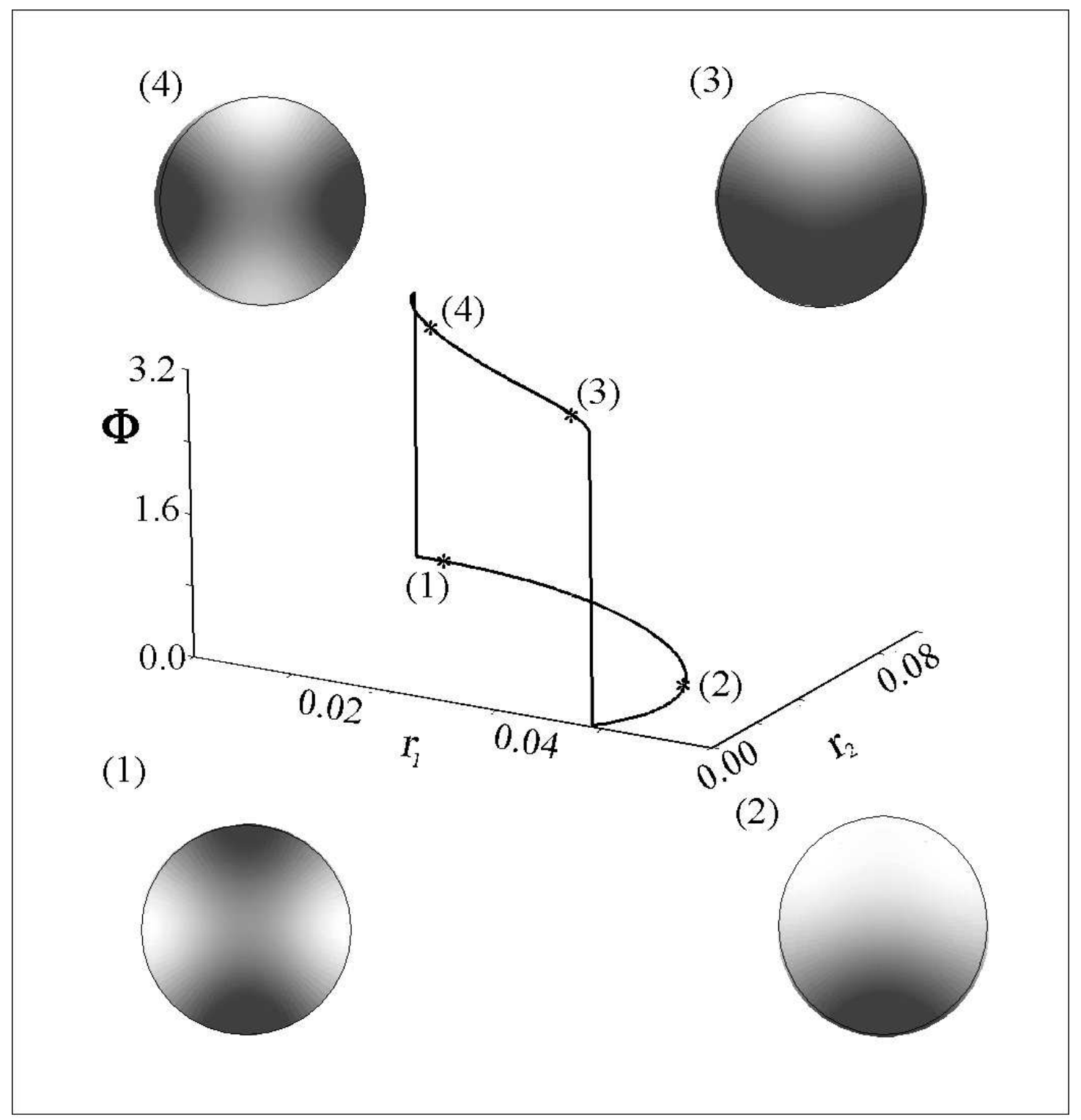

Figure 6a. 


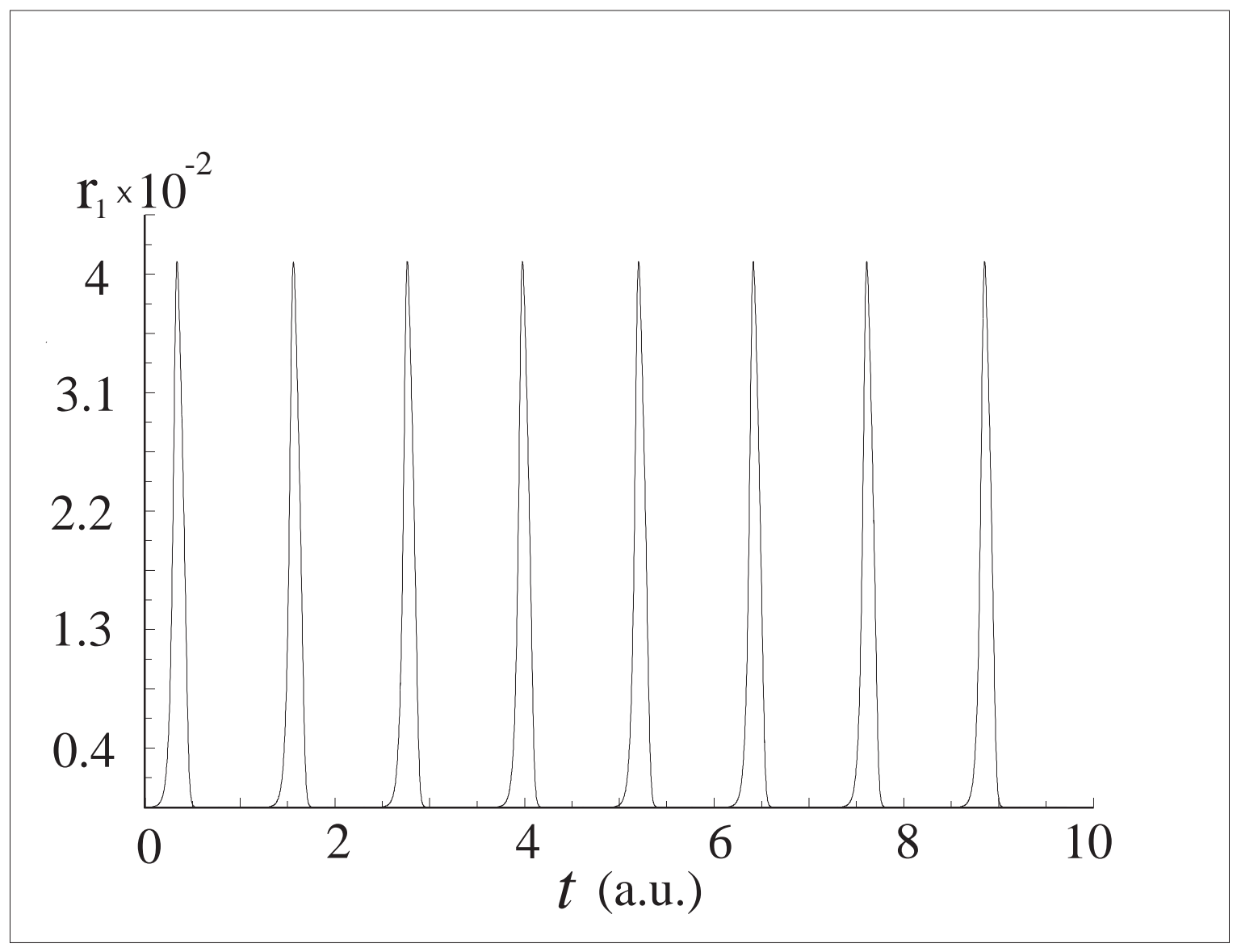

Figure $6 b$. 


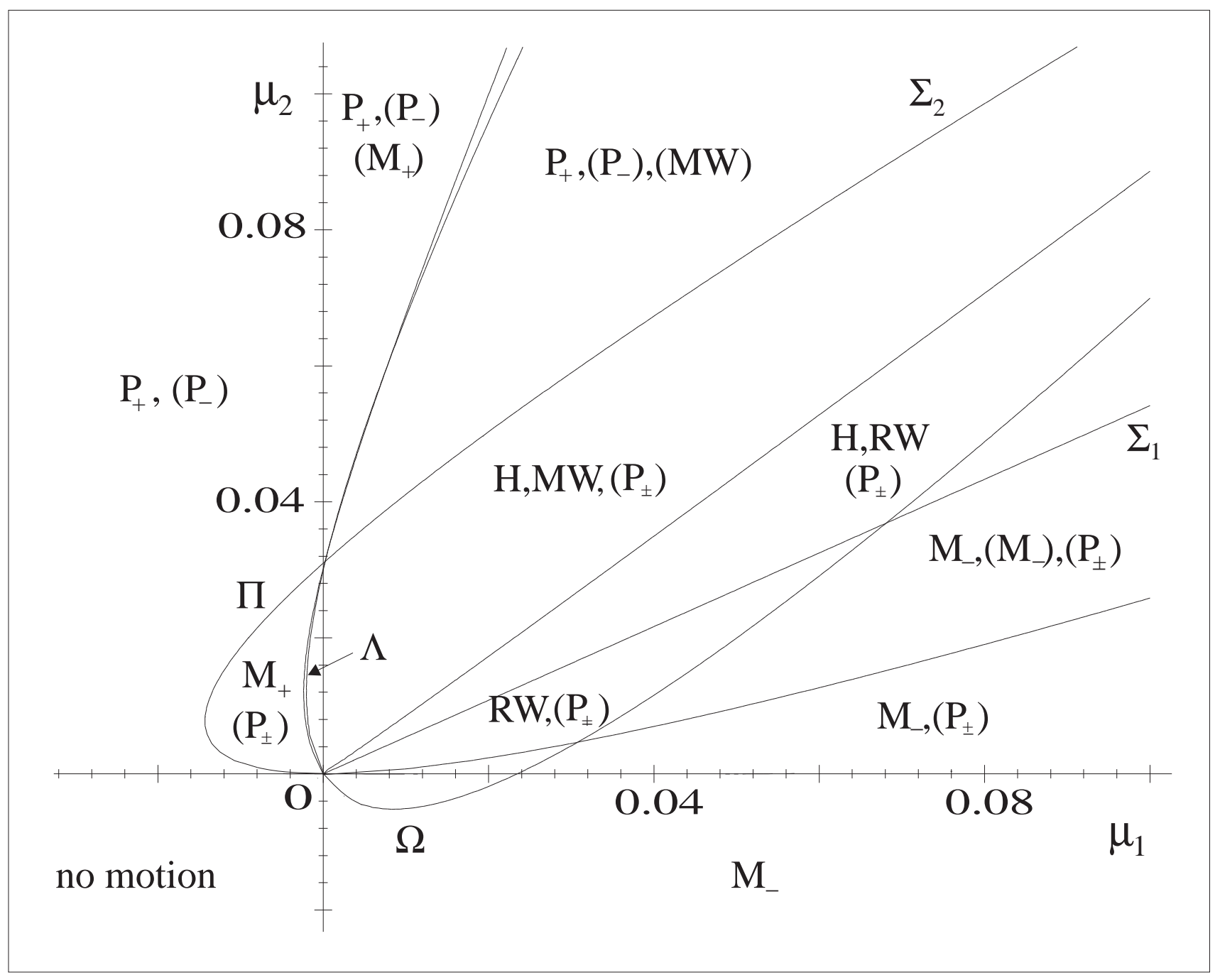

Figure 7. 


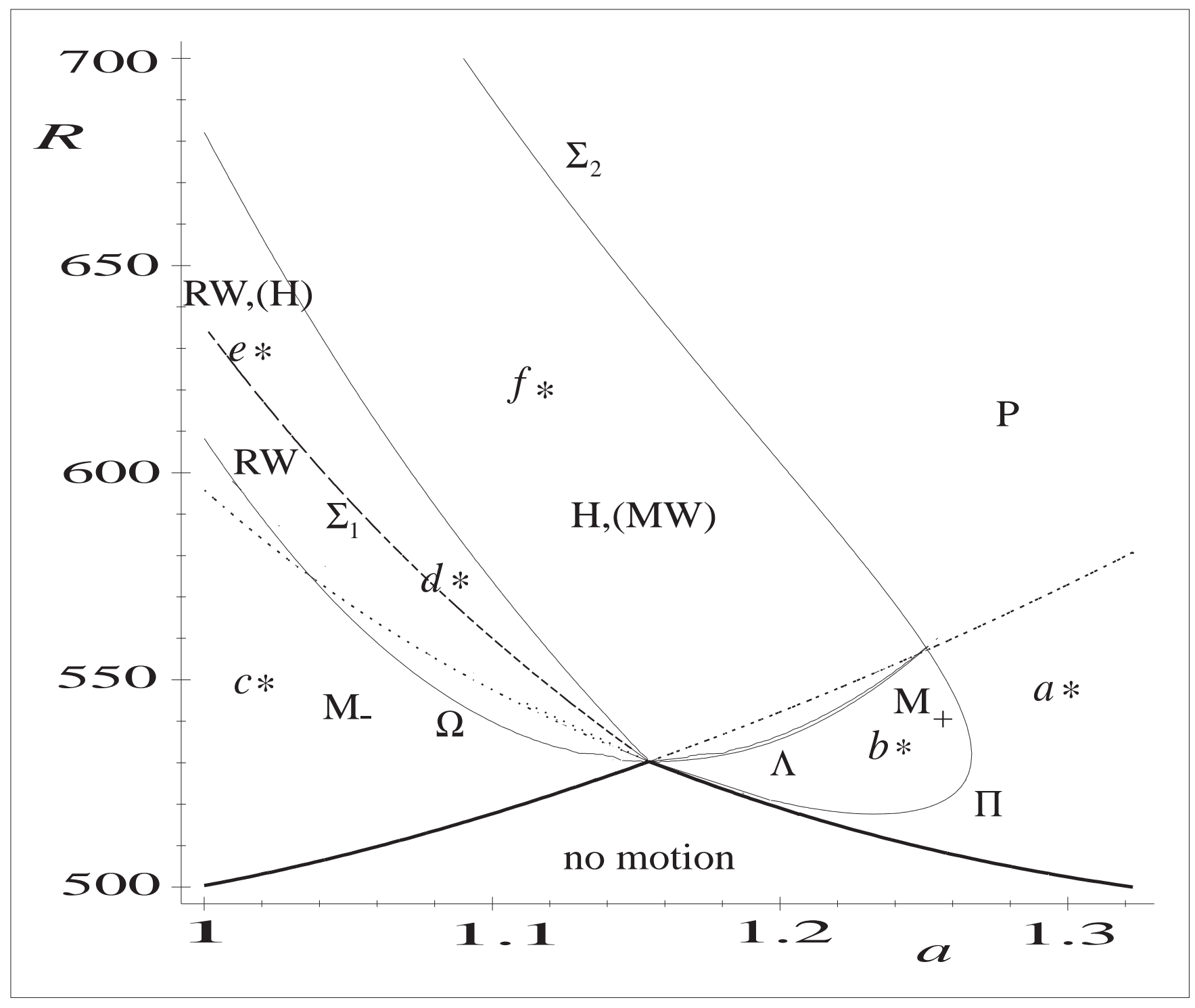

Figure 8. 


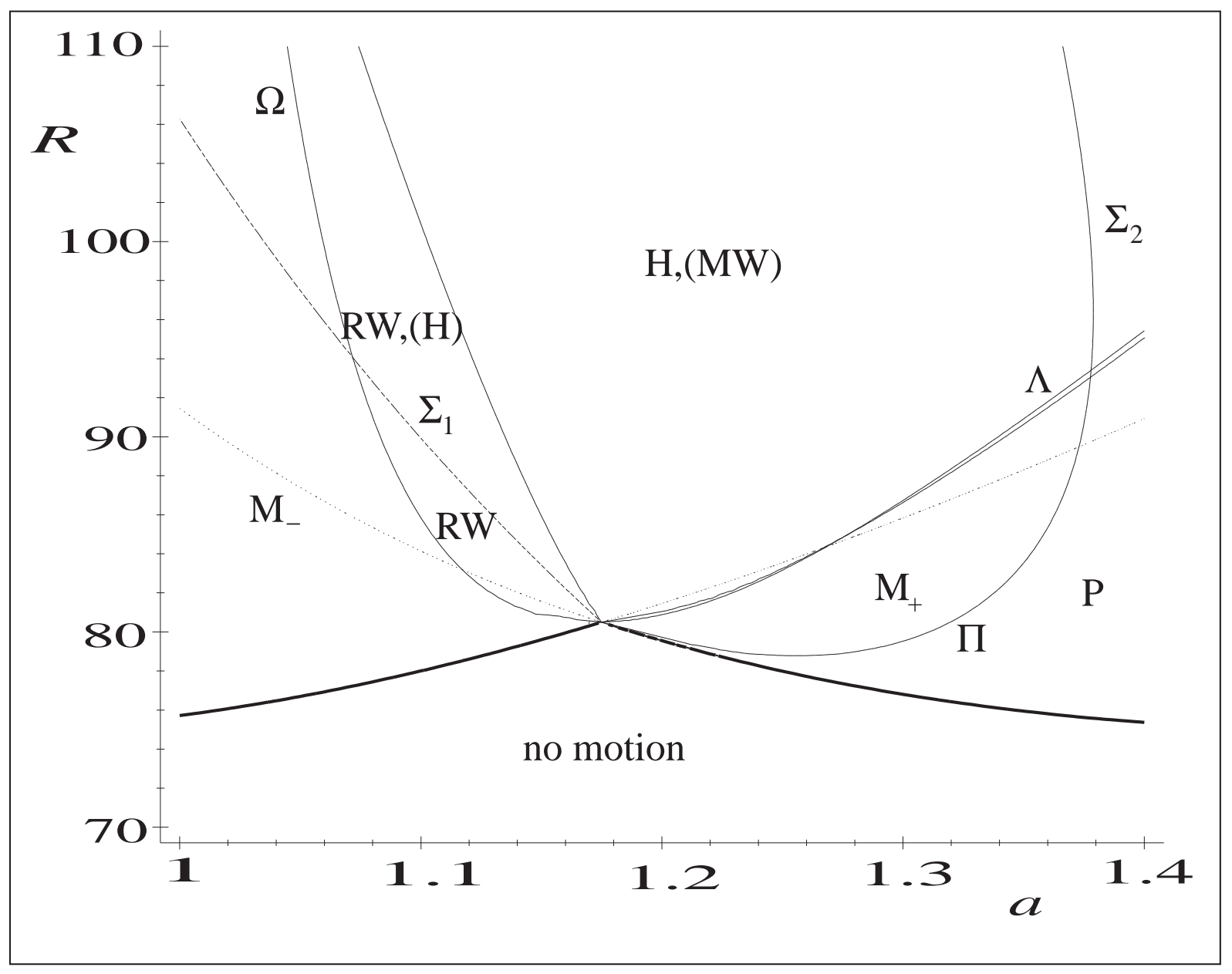

Figure 9a. 


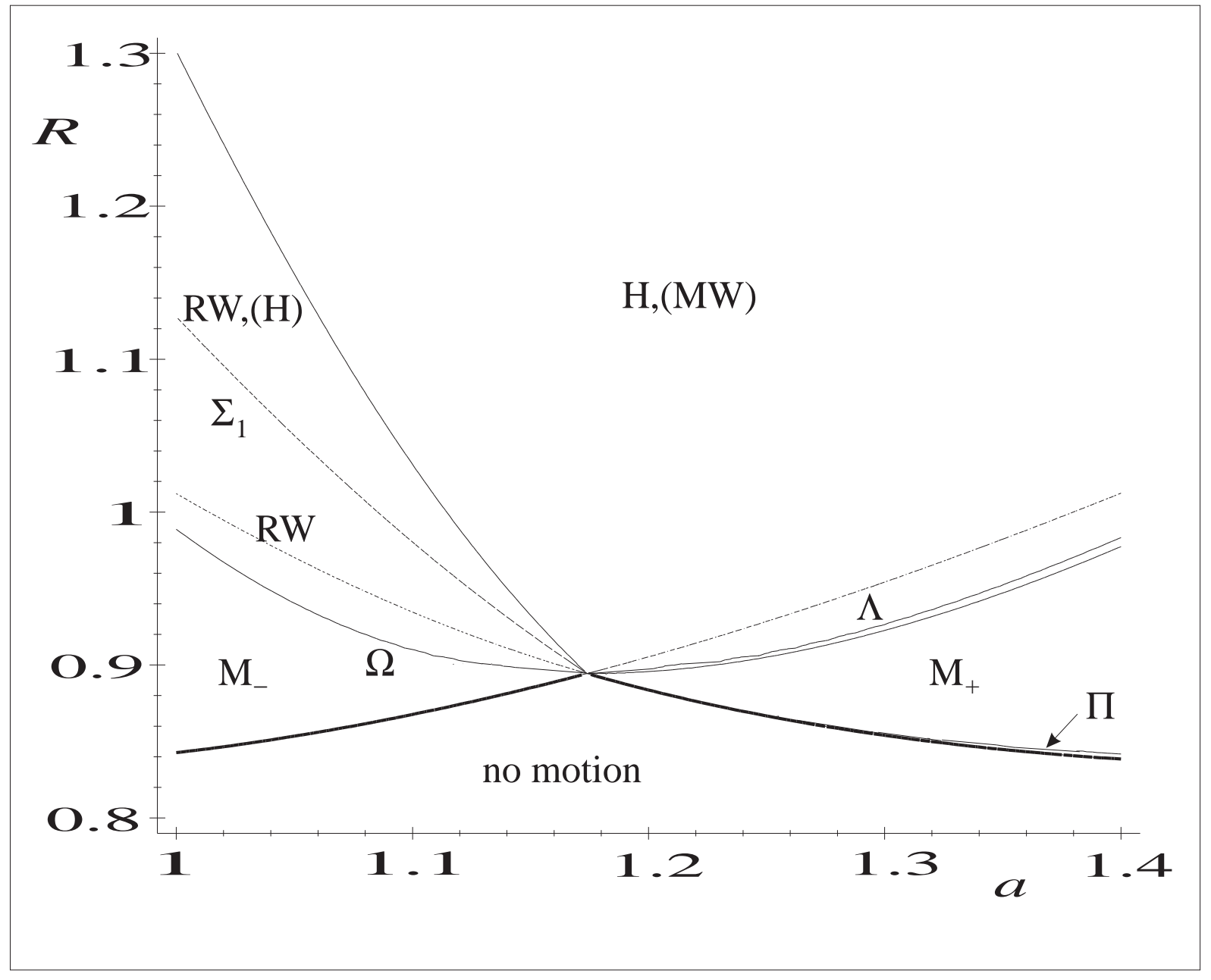

Figure 9b. 